\title{
Amorphous alloys formed by microsecond current puises
}

\author{
B. M. Clemens \\ Physics Department, General Motors Research, Warren, Michigan 48090-9055 \\ R. M. Gilgenbach and S. Bidwell \\ Nuclear Engineering Department, The University of Michigan, Ann Arbor, Michigan 48109
}

\begin{abstract}
(Received 6 November 1986; accepted for publication 22 December 1986)
We report the use of microsecond current pulses to transform layered crystallne nickelzirconium fims to amorphous alloy. Starting material was electron beam deposited multilayers with a composition modulation wavelength of $34 \mathrm{~nm}$, an average composition of $\mathrm{Ni}_{63} \mathrm{ZF}_{37}$, and a total thickness of $680 \mathrm{~mm}$. Electrical pulses were approximately rectangular and about $3 \mu \mathrm{s}$ in duration, with an intensity of several hundred amperes, directly coupling 1.6 to $3 \mathrm{~J}$ of energy uniformly into the film. By monitoring current and volkage, the reaction and melting of the sample were observed, and the total energy of the pulse was easily computed. A sharp threshold in pulse energy for sample transformation was observed. A simple heat fow calculation demonstrated that the chemical energy released by the reaction, and the change in diffusion kinetics as the sample temperature exceeded the glass transition temperature of the amorphous alloy, are responsible for this sudden onset. The maximum temperature estimated from this calcuiation is below the melting point of the constituents, and the cooling rate is $10^{7}-$ $10^{8} \mathrm{~K} / \mathrm{s}$ which is in agreement with the formation of amorphous alloy.
\end{abstract}

Since the early work of Klement et al., ${ }^{1}$ interest has focused on production of metastable alloys by rapid quenching from the melt. ${ }^{2}$ We recentiy reported the use of electrical current pulses to thermally treat metal films, including the first use of this technique to produce amorphous alloys by rapid quenching. ${ }^{3}$ Electrical pulses of several microseconds duration produce meiting of zirconium-nickel multilayers on sapphire substrates. The subsequent cooling rate is sufficient $\left(10^{7}-10^{8} \mathrm{~K} / \mathrm{s}\right)$ to bypass crystailization. Furthermore, the large negative heat of mixing between the constifuents results in a sharp threshold in pulse power required to produce melting.

Electrical pulses have several attractive features for rapid thermal treatment of metal films. The energy is deposised uniformly and coupled directly to the film. The pulse shape can be manipulated to give the desired temporal behavior over several orders of magnitude. By monitoring the voliage and current, the sample resistance can easily be monitored, so phase changes can be directly observed, and energy deposited can be easily computed. This provides this method with a buitt-in diagnostic unavailable with other techniques.

The nickei-zirconium system was chosen for this study for a variety of reasons. The amorphous phase readily forms by conventional liquid quenching over a wide composition range, and its properties have been extensively studied. ${ }^{4}$ Solid state reaction during long time anneals of multilayered nickel-zirconium structures also results in amorphous phase formation, and investigations of this process on a fast time scale are of fundamental interest in understanding the role of nucleation of the competing phases. There is a large negative heat of mixing between the constituents in this system, with the result that a multilayered structure contains substantial chemical energy. This results in a self-sustained reaction during thermal treatment on the microsecond time scale.

Nickel-zirconium muitilayer samples were prepared by rotating the sapphire substrates above shielded electron beam evaporation sources in à vacuum system with a base pressure below $10^{-8}$ Torr. The rates were set to produce an average composition of $\mathrm{Ni}_{63} \mathrm{Z}_{37}$ and the rotary motion was set to produce a composition modulation wavelength of 34 nm. Twenty bilayers were deposited resuiting in a total thickness of $680 \mathrm{~mm}$. X-ray diffraction was performed with $\mathrm{Cu} K_{\alpha}$ in thin-fim Seenan-Bohlin geometry. Auger depth profiling was performed on selected samples before and after pulsing to determine the degree of mixing and the level of impurities.

Samples were heated by a pulsed discharge circuit consisting of a multistage artificial transmission line. Currents in the sample and a parallel resistor were inductively measured by current transformers. The ends of the sample were pressed onto copper tape electrodes on a glass piate. Samples were puised in air or in argon at atmospheric pressure.

The structure of the starting material was polycrystalline elements as shown by the x-ray diffraction in Fig. 1 (a). Pulses with an energy of $2.3 \mathrm{~J}$ or greater resulted in amorphous phase formation. Figure $1(b)$ shows $x$-ray diffraction for a sample puised with $2.3 \mathrm{~J}$ electrical energy input. The nickel and zirconium crystalline peaks are no longer evident, and a broad band characteristic of amorphous material has appeared. There is also a weak peak which can be identified as zirconium oxide, which Auger depth profiing shows to be confined to the top $15 \mathrm{~nm}$ of the surface region. This oxide peak is more pronounced in samples pulsed in air than in those pulsed in Ar, indicating that the oxidation is occurring curing puising. Auger depth pronling also showed that the initial strong composition modulation was completely destroyed by the puise. The surface of the sample both before and after pulsing is mostly smooth and featureless as seen by scanning electron microscopy. However, after pulsing there are some pinholes with smooth, rounded edges which presumably are the result of defects or dust introduced during sample handing prior to deposition which are blown away 


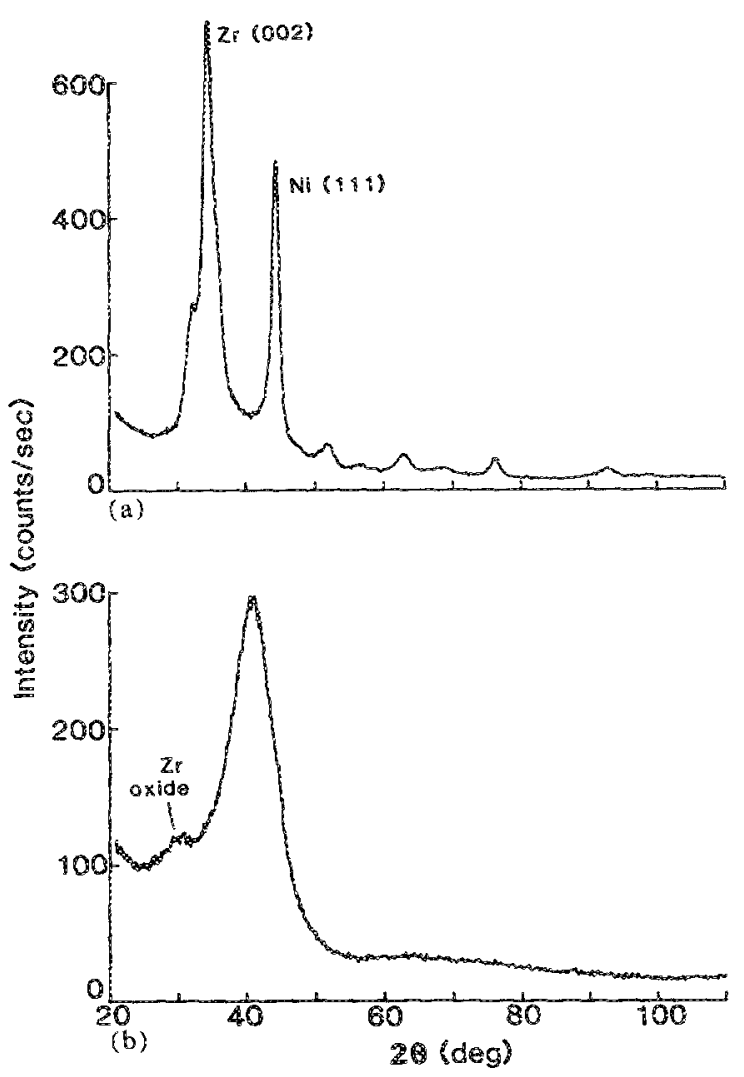

FIG. 1. Seeman-Bohlin $x$-ray difraction for nickel-zirconium samples (a) before and (b) after $2.3 \mathrm{~J}$ current pulse.

by the puise. These rounded edges and some wrinkling near the contact area are evidence that the sample was melted by the pulse.

The melting transition can be clearly observed in the current and voltage traces diring the pulse. Shown in Figs. 2 (a) and 2 (b) are the voltage and current traces for a sample pulsed with $2.9 \mathrm{~J}$. The rapid rise in resistance which accompanies mixing and melting of the layers results in a sharp rise in sample voltage and a drop in current which occurs between about 1.8 and $2.3 \mu \mathrm{s}$. This corresponds to about a $40-60 \%$ increase in sample resistivity. The position of this

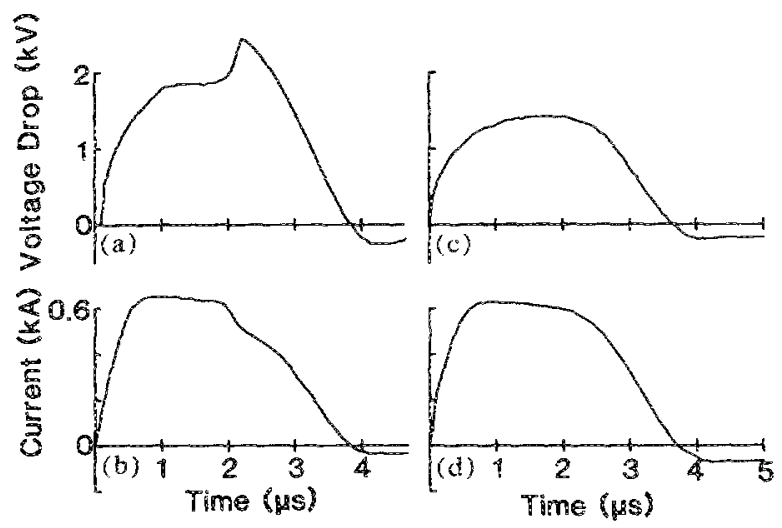

FIC. 2. Voltage and current traces for current pulses: (a) voltage for $2.9 \mathrm{I}$ pulse, (b) current for $2.9 \mathrm{I}$ pulse, (c) voltage for $2.0 \mathrm{I}$ pulse, and (d) currene for $2.0 \mathrm{~J}$ pulse. transition is determined by the pulse power with lower pulse powers resulting in a later transition. Pulses with an energy of $2 \mathrm{I}$ or less produced no transition as shown in Figs. 2(c) and 2 (d) which show voltage and current traces for a $2-J$ pulse. A pulse energy of $2.16 \mathrm{~J}$ produced a small (12\%), rapid rise in resistance.

Formation of amorphous phase material occurred at the same pulse energies as the resistance transition. Shown in Fig. 3 is the fraction of crystalline material remaining, extracted from the $x$-ray diffaction data, plotted versus pulse energy. A sharp drop occurs between 2 and $2.3 \mathrm{~J}$, which is where the resistive transition begins to appear. An increase of $15 \%$ in pulse power in going from 2 to $2.3 \mathrm{~J}$ results in a decrease in crystalline peak intensity from $97 \%$ to $4 \%$.

Several physical phenomena are occurring as the sample heats during the palse. Extrapolation of low-temperature data" reveals that solid state fornation of an amorphous alloy will begin to occur on the microsecond time scale at a temperature of about $1000 \mathrm{~K}$, well below the melting point of the constituents. This reaction will release the substantial heat of mixing which will cause a further temperature increase, resulting in a self-sustained reaction. Furthernore, the diffusion kinetics in the reacted amorphous product will change in nature as the glass transition temperature $\left(T_{g}\right)$ of the amorphous phase is exceded. Diffusion in the solid state follows an Arrhenius form while difusivity in liquid metals follows the Fukner-Vogel form,

$$
D=D_{0} \exp \left[-B /\left(T-T_{0}\right)\right]
$$

In equilibrium, the crossover between these two regines will occur at $T_{g}$, which for amorphous nickel-zirconium depends on composition and is between 600 and $850 \mathrm{~K}{ }^{4}$ In conditions of rapid heating, the crossover temperature will occur wher the atomic jump frequency becomes comparable to the time scale of the experiment. ${ }^{6}$ Using the difiusivity from lowtemperature solid state reaction studies, "we find a crossover temperature of $900 \mathrm{~K}$.

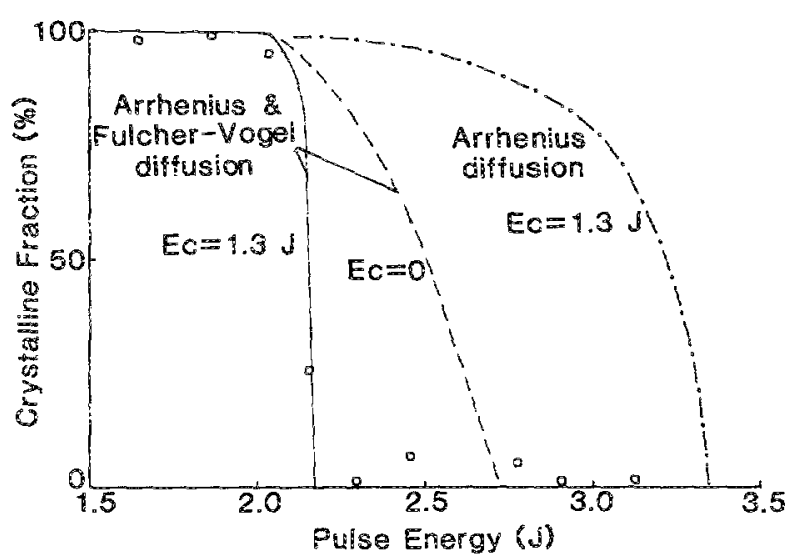

FIG. 3. Volume fraction of sumple remaining crystalline after pulse vs pulse energy. Circles are derived from experimental $x$-ray data; solid line is the results of a model calculation which includes a chemical driving term and both Arrhenius and Fulcher-Vogel behavior for the diffusivity. Dashed line shows the results of the model with no chemical driving term, and dotdashed line shows the results of the model with a chemical driving but only Arrhenius behavior for the diffusivity. 
In order to gain insight into the temporal belavior of the sample temperature during these puises, and to understand the effects of the exothermic reaction between the constitnents and the change in diffusion kinetics above $T_{g}$, a simple heat flow calculation was performed. We used the Green's function solution to the heat diffusion equation, ${ }^{7}$ assuming the only impediment to heat fow was the finite thermal diffusivity of the substrate. The thermal driving function was taken to be the sum of the measured electrical power and a chemical power term, divided by the heat capacity of the film (taken from data for the elements in the literature). ${ }^{8}$ The magnitude of the chemical energy term was calculated by the method of Miediema ${ }^{9}$ to be $1.3 \mathrm{~J}$, which is a substantial fraction of the clectrical pulse energy of about 23 . The rate of chemical energy release is proportional to the growth rate of the interfacial alloy, calculated from the square root of time growth law. For temperatures below the diffusion crossover temperature, the diffusivity $D$ followed an Arhenius form with prefactor and activation energy taken from solid state reaction data. ${ }^{5}$ Above the crossover temperature the Fulcher-Vogel form [Eq. (1)] was used for the diffusivity, with rough estimates for the parameters selected in a mamer similar to that used by Spaeper and Lin. ${ }^{10} B$ was taken to be $1300 \mathrm{~K}, T_{0}$ and $D_{0}$ were calculated using the Stokes-Einstein relation between the viscosity and diffusivity, and assiming that the viscosity is $10^{12}$ Poise at $T_{\mathrm{g}}(700 \mathrm{~K}),{ }^{4}$ and $10^{-2}$ at the melting point of the alloy $(1550 \mathrm{~K}){ }^{4}$ The thermal diffusivity of the substrate was used as an adjustable parameter so the model calculation produced reaction at the observed pulse input energy. The value found in this manner 10.036 $\mathrm{cm}^{2} / \mathrm{s}$ ) is in good agreement with published data for this temperature range, "indicating that the assumption of ininite thermal conductivity of the sample-substrate interface is reasonable.

For pulses with energy greater than $2.3 \mathrm{~J}$ this model predicts a sharp rise in sample temperature due to the influence of chemical energy. This is reminiscent of the sharp rise in sample resistance observed in these pulses. The self-sustained reaction produces a sharp threshold in pulse energy for transformation as shown in Fig. 3, where the fraction remaining crystalline is plotted versus puise energy for the model calculation both with and without the chemical driving term. Also shown are the results of the modei for the case where the difusivity follows an Arthenius form throughout the temperature range. The reaction temperatures predicted by this model are around $1000 \mathrm{~K}$, which is above $T_{g}$ for the amorphous phase, so this is not a solid state reaction, but a reaction taking place with the product in an undercooled liquid state.

An estimate of the cooling rate can be calculated from this model by dividing the diference between the raximum temperature and $T_{g}$ by the time it took the sample to cool over this range. This is about $10^{7}-10^{8} \mathrm{~K} / \mathrm{s}$, which is in good agreement with the observation of amorphous phase formation. The heating rate found from this calculation is about $4 \times 10^{8} \mathrm{~K} / \mathrm{s}$.

Electric current pulses offer the ability to thermally treat films on a microsecond time scale. This technique has several advantages over conventional techniques including a built-in diagnostic which allows calculation of deposited energy and sample resistance. We have used this technique to produce amorphous nickel-zirconium from layered crystalline film. A simple model calculation demonstrates the role of chemical energy which in this system induces a self-sustained reaction. The transformation from layered crystaline elements to amorphous alloy does not involve melting of the constituents, but oceurs above the glass transition temperature of the amorphous alloy and below the melting point of the crystalline phases.

We would like to acknowledge the support of $J$. C. Buchholz in the initial stages of this project. We also would like to acknowledge helpful conversations with W. L. Johnson and $P$. Spaepen. Auger depth profiling was performed by A. Dow and S. Simco of the Analytical Chemistry Depart. ment of General Motors Research.

\footnotetext{
${ }^{1}$ W. Kiement, R. H. Willens, and R. Duwet, Nature 387,869 (1960). ${ }^{2}$ Rapidly Quichiched Metals, Procedings of the Fifth Intemational Conference on Rapidly Quenched Metals. Wurburg, Gemanys September 1984, edited by $\mathrm{S}$. Steeb and H. Warlimont (North-Holland, Amsterdam, 1985).

'B. M. Clemens, J. C. Buchholz, R. M. Gilgenbach, and S. Bidwell, Iroceedings of The Materiais Research Sociery, Tumbull Symposium, 1985 (in press).

${ }^{4} Z$. Altounian, $T u$ Guohua, and J. O. Strom-Olsen, J. Appl. Phys. 54, 3111 $(1983)$.

B. M. Clemens, W. L. Johnson, and R. B. Schwarz, J. Non-Cryst. Solids 61262,817 (1994); B. M. Clemens, Phys Rev. B 33, 7615 (1986). "fi. Spaepen (private commanication).

E. Butkov, hathematical Physics (Addison-Wesley, Reading, Massachusetts, 1968), p. 526.

"CRC Handbook of Chomisny and Physics, 55 th ed., R. C. Weast, ed. (CRC. Cleveland, Ohio, 1975).

"A. R. Miedema, Philips Tech. Rev, 36, 217 (1976).

"F. Spaepen and C. J. Lin, in Amorphous Metals and Non-Fguilibrium Processing, Materials Research Society Procedings, edited by M. won Almen (Ges Editions de Thysique, les Ulis, France, 1984), p. 65.

"Y.S. Touloukian and C. Y. Ho, cds., Thermophysical Properties of Matter (IFl/Plenun, New York, 1970), Voi. 2, p. 93 and Vol. 5, pp. 24.25.
} 